\title{
FACTORES DETERMINANTES DEL DESEMPEÑO FINANCIERO EN EL SECTOR MANUFACTURERO EN LA REPÚBLICA DEL ECUADOR
}

Germán Marcelo Salazar-Mosquera

Universidad Técnica de Ambato

Ecuador 
Panorama Económico, Vol. 25 - No. 2 (Abril - Junio de 2017), pp. 243-254

Germán Marcelo Salazar-Mosquera

Factores determinantes del desempeño financiero en el sector manufacturero en la República del Ecuador

\title{
Resumen
}

El objetivo de este estudio es identificar los factores que inciden en la rentabilidad de las sociedades manufactureras con base en la información suministrada por la Superintendencia de Compañías. El análisis de correlación permite concluir que la rotación de ventas y la rentabilidad sobre el activo son los factores determinantes en la rentabilidad financiera, es decir, un incremento en los indicadores señalados, implica un incremento en la rentabilidad financiera de las empresas manufactureras en Ecuador.

Palabras clave: Desempeño financiero; Indicadores Financieros; Rentabilidad.

Clasificación JEL: D52, E44, D57, D92

Determinant factors of financial perfomance in the manufacturing sector in the Republic of Equator

\begin{abstract}
This research identifyes the factors that affect profitability of manufacturing firms based on the information provided by the National Superintendence of Firms. Correlation analysis indicates that sales and asset's profitability are the main drivers of financial profits. In this sense, augmenting these variables will enhance profit-earning capacity of manufacturing firms in the Republic of Equador.

Keywords: Profitability, financial indicators, financial performance
\end{abstract}

JEL Classification: D52, E44, D57, D92

Facteurs déterminants de la performance financière dans le secteur manufacturier en République de l'Équateur

\section{Résumé}

Cette recherche identifie les facteurs qui affectent la rentabilité des entreprises manufacturières sur la base des informations fournies par la Surintendance nationale des entreprises. L'analyse de corrélation indique que les ventes et la rentabilité des actifs sont les principaux moteurs des bénéfices financiers. En ce sens, l'augmentation de ces variables augmentera la capacité de profit des entreprises manufacturières de la République de l'Équateur.

Mots-clés: Rentabilité, indicateurs financiers, performance financière.

Nomenclature JEL: D52, E44, D57, D92 


\section{Factores determinantes del desempeño financiero en el sector manufacturero en la República del Ecuador}

INFORMACIÓN DEL ARTíCULO

Recepción de artículo: 06/01/2017

Concepto de evaluación: 09/02/2017

Aceptación de artículo: 28/03/2017
Germán Marcelo Salazar-Mosquera* Universidad Técnica de Ambato Ecuador

\section{INTRODUCCIÓN}

La economía real de un país se sustenta en la producción de bienes y servicios. Los beneficios de su crecimiento se reparten entre todos sus actores (Mazzei, 2016). Los estudios principales que relacionan la economía real con la economía financiera son: "Los flujos del comercio internacional" (Ohlin, 1933) y "La identificación de ventajas comparativas entre los países" (Leontief, 1953)

El desarrollo de la economía real se sustenta en las teorías de ciclos económicos (Schumpeter, 1935), de las ondas largas de la vida económica (Kondratieff, 1922), y las innovaciones tecnológicas como motor de ciclo económicos, (Freeman, 1998). La economía real de un país experimenta cambios dramáticos por el entorno externo y por el relevo incesante de los gestores financieros y económicos de las naciones.

El sector manufacturero constituye uno de los actores importantes de la economía real porque, según datos del Banco Mundial, el valor agregado de la industria a precios actuales en el año 2014 representaba el 27,61\% del PIB mundial, mientras que para el año 2015 descendió al 27,02\%. Además que dinamiza los otros sectores como el comercio y los servicios.

La misma fuente de datos nos permite calcular, el porcentaje de participación del valor agregado de la industria a precios actuales, en el 2014, representaba el 37,68\% del PIB en el 2014, mientras que para el año 2015, descendió al 31,87\%.

\footnotetext{
* Autor para correspondencia

Correos electrónicos: gm.salazar@uta.edu.ec*
} 
El entorno actual caracterizado por procesos sistemáticos innovación tecnológica e información globalizada e inmediata de las tendencias económicas, exige a los administradores de las empresas investigar y aplicar las alternativas para gestionar en forma eficiente y eficaz los recursos monetarios, humanos y materiales. Adoptando procesos de toma de decisiones que permitan cumplir en un porcentaje aceptable los objetivos y metas empresariales relacionados con las principales variables financieras de liquidez y rentabilidad.

En el siglo XXI las principales fuentes de empleo y el sustento del desarrollo económico de la Provincia del Tungurahua hasidoel sector manufacturero, sinembargo, la baja competitividad, la baja rotación de activos y la administración inadecuada de las fuentes de financiación han influido para que se convierta en un sector poco rentable y se haya detenido su crecimiento.

En la última década no se encuentran estudios realizados que identifiquen los factores que inciden en la rentabilidad de las empresas del sector y que permitan tener información relevante para la formulación de planes estratégicos, con la finalidad de mejorar la productividad y fortalecer la competitividad. Sin embargo, a nivel internacional, el desempeño financiero ha sido objeto de algunos estudios científicos.

Es necesario que las empresas ubicadas en los distintos sectores evalúen constantemente la evolución de sus estructuras e indicadores financieros y realicen su planeación organizacional, tendiente a mantener un adecuado equilibrio, pues como se presentó a lo largo del trabajo, los estados e indicadores financieros dejan en evidencia el resultado de las decisiones tomadas al interior de estos sectores, así como el im- pacto en su capacidad para competir en un mercado, cada vez más internacionalizado (Castaño y Arias, 2014).

El factor clave que explica el comportamiento de la eficiencia en las innovadoras (empresas) es la rotación de los activos operacionales, y más específicamente la rotación de inventarios, mientras que la eficacia en las no innovadoras se concentra en que logra un mayor margen bruto, resultado de unas ventas en crecimiento constante y unos costos fijos inferiores (por su menor inversión en activos) (Rivera y Ruiz, 2011).

Esta optimización debe partir de buscar la excelencia empresarial y utilizar la comparación con los mejores, las empresas de clase mundial, para aprender de ellas buscando cerrar las brechas y lograr los altísimos niveles de competitividad que caracterizan el mundo actual (Medina, 2010)

El objetivo de la presente investigación es identificar los factores determinantes del componente principal del desempeño financiero que es la rentabilidad en las compañías manufactureras de la provincia del Tungurahua, utilizando los indicadores proporcionados por la superintendencia de compañías.

La investigación debe responder a la pregunta ¿Cuáles son los factores determinantes de la rentabilidad en el sector manufacturero de la provincia del Tungurahua?

Se justifica la presente investigación porque la identificación de los factores determinantes de la rentabilidad permitirá a las empresas del sector, realizar una planificación adecuada de sus recursos y lograr un crecimiento sostenible $\mathrm{y}$ sustentable. 
La evaluación se efectúa expost y permite retroalimentar el análisis. En los años posteriores de debe básicamente, comparar los resultados reales con los resultados de la presente investigación. El objetivo final de la evaluación recurrente será mejorar el desempeño financiero de las compañías manufactureras de la provincia del Tungurahua.

\section{FUNDAMENTO TEÓRICO}

La información sobre el desempeño de la empresa, en particular su rentabilidad, se requiere con el fin de valorar los cambios potenciales en los recursos económicos que sea probable se controlen en el futuro. En este sentido es importante la información sobre la variabilidad del desempeño. La información sobre el desempeño es útil para predecir la capacidad de la empresa para generar flujos de efectivo a partir de su base existente de recursos. También es útil para la formación de los juicios sobre la efectividad con la cual la empresa puede emplear recursos adicionales (IASC, 1989).

Para Robles, (2012) El análisis e interpretación de los estados financieros es básico para las empresas, ya que implica una evaluación de la información financiera; de este modo, se analiza detalladamente cómo se desarrollan estos aspectos, y los resultados se obtienen de manera cuantitativa. Rivera y Ruíz, (2011), describen las fuentes de datos para realizar un diagnóstico del desempeño financiero e identifican la existencia de tres metodologías, cuyo análisis se efectúa a partir de: a) información contable, b) información del mercado de valores, y c) criterios de valor añadido.

Castillo y García, (2012) realizan un análisis de los factores explicativos de la rentabilidad de las empresas vinícolas de Castilla-La Mancha. Mediantela aplicación de un modelo econométrico cuya variable dependiente es la rentabilidad y entre las variables independientes se incluyen otros ratios financieros. De los resultados obtenidos infieren que la rentabilidad de las empresas proviene entre otros de su estructura financiera (Mayor rentabilidad si existe un mayor porcentaje de recursos propios y mejor liquidez)

El análisis financiero basado en la información contable es el más utilizado por su amplia difusión, sin embargo, tiene sus propias limitaciones derivadas de las inconsistencias de los estados financieros. El análisis basado en los datos suministrados por el mercado de valores tiene su utilidad en economías de bursatilidad alta. El análisis fundamentado en el valor añadido, ha sido muy poco difundido.

La presente investigación se basa en la información contable que está contenida en los cuatro estados financieros básicos: Estado de situación financiera, Estado de resultados del periodo y otros resultados integrales, Estado de flujos de efectivo y el Estado de cambios en el patrimonio.

Para analizar e interpretar los estados financieros, existen tres métodos tradicionales: Análisis Vertical, Análisis horizontal y el uso de indicadores financieros.

Análisis Horizontal: también denominado análisis comparativo. "Los analistas realizan un análisis de los estados financieros comparativos revisando los balances, las declaraciones de ingresos, o las declaraciones del flujo de efectivo consecutivos de un periodo a otro. Esto por lo común implica la revisión de los cambios de las cuentas de los balances individuales en un intervalo de uno o varios años. La información más importante que a menudo revela el análi- 
sis de estados financieros comparativos es la tendencia". (Wild, Subramanyam y Halsey, 2007). Luego de comparar las cifras de los estados financieros de un periodo con otro se obtienen variaciones absolutas y relativas. Este análisis se convierte en una herramienta útil cuando se identifican las causas raíces de las variaciones, lo cual constituye un insumo importante para mejorar la calidad de la toma de decisiones.

Análisis vertical: "Consiste en tomar un estado financiero y relacionar cada una de sus partes con un total determinado dentro del mismo estado, el cual se denomina cifra base" (Estupiñan y Estupiñan, 2006), Para obtener un beneficio de este análisis se debe comparar con los líderes del sector industrial lo cual nos permitirá orientar las decisiones hacia una estructura adecuada de inversión y financiamiento.

Según Van Horne y Wachowicz, (2010), "Las herramientas utilizadas para evaluar la condición financiera y el desempeño de la empresa son las razones financieras" y añaden; "El analista financiero usa estos indicadores casi como un médico hábil interpreta los análisis de laboratorio. En combinación, y a través del tiempo, estos datos ofrecen un panorama valioso de la salud de la empresa: su condición financiera y su rentabilidad". Para que la interpretación de los resultados de este análisis logre el objetivo de contribuir a mejorar la toma las decisiones es necesario realizar un análisis integral porque cada una de las cifras de los indicadores por si solas no presentan una imagen fiel del sujeto de estudio.

Para analizar la situación financiera de las empresas, se utilizan un gran número de indicadores financieros, que se dividen en cuatro grupos: Indicadores que miden la liquidez, Indicadores que miden el endeudamiento, Indicadores que miden la rentabilidad e indicadores que miden la rentabilidad.

En la presente investigación relacionada con identificar los factores determinantes en el desempeño empresarial, se utilizan los siguientes indicadores:

1. Liquidez-corriente, que se obtiene de dividir el total de activos corrientes entre el total de pasivos corrientes e indica la cantidad de unidades monetarias que la empresa, dispone, en promedio para cubrir cada dólar del pasivo corriente. Es necesario advertir que este indicador no garantiza la sincronización entre entradas y salidas de efectivo.

2. Prueba ácida. Resulta de dividir el total del activo corriente menos los inventarios entre el pasivo corriente $y$ mide la capacidad mediata de pago de las obligaciones a corto plazo con los activos líquidos.

3. Endeudamiento sobre el activo. Se obtiene de la división del pasivo total entre el activo total, también denominado índice solidez. Permite visualizar la porción de los activos que se encuentran financiados con recursos ajenos.

4. Endeudamiento sobre el patrimonio. Es el resultado de la división entre el pasivo total y el patrimonio total $\mathrm{y}$ permite calcular el número de veces que el patrimonio de la empresa está comprometido con los acreedores.

5. Endeudamiento sobre el activo fijo neto. Se obtiene de la división entre el pasivo total y el activo fijo neto, y permite calcular la porción del activo fijo financiada con recursos de los acreedores.

6. Apalancamiento operativo. Es el resultado de la división de las ventas totales menos los costos variables 
totales entre las ventas totales menos los costos variables totales menos los costos fijos totales y permite calcular la variación porcentual que se produce en la utilidad operativa como efecto de una variación porcentual en la cifra de ventas.

7. Apalancamiento financiero. Se obtiene dividiendo la utilidad operativa entre la utilidad operativa menos los gastos financieros y permite medir el efecto que produce la utilidad neta como consecuencia de una variación relativa en la utilidad operativa, cuando se utilizan recursos ajenos con costo para financiar el capital de trabajo y los activos fijos.

8. Rotación de cartera. Mide el número de veces que rotan las cuentas por cobrar comerciales en un ejercicio económico. Es el resultado de la división entre las ventas netas a crédito entre el promedio de cuentas por cobrar.

9. Rotación del activo fijo. Se calcula dividiendo las ventas netas a entre el activo fijo neto de depreciaciones y permite visualizar el número de veces que rota el activo fijo en un periodo económico.

10. Rotación de ventas. Es el resultado de la división entre las ventas netas y el activo total. Permite calcular el número de veces que rota la inversión total en un año.

11. Rentabilidad neta sobre el activo. Se obtiene dividiendo la utilidad neta después de intereses, participación laboral e impuestos entre el activo total y permite calcular el porcentaje de productividad del activo total.

12. Margen bruto en ventas. Se obtiene de dividir la utilidad en ventas entre las ventas netas totales. Su resultado nos indica el porcentaje de ganancia que se separa la empresa de los ingresos por ventas.
13. Rentabilidad sobre el patrimonio. Es el resultado de la división de la utilidad neta entre el patrimonio y permite calcular el porcentaje de ganancia que la empresa proporciona a sus socios o accionistas en relación a la inversión de los recursos económicos que han efectuado.

Los indicadores seleccionados permiten obtener el coeficiente de correlación entre cada uno de los indicadores obtenidos por las empresas con el indicador más representativo del desempeño empresarial que es la rentabilidad sobre el activo.

\section{MATERIALES Y MÉTODOS}

Una vez que se ha establecido el objetivo de la investigación que es el de identificar los factores determinantes en la rentabilidad de las empresas manufactureras de la provincia del Tungurahua, se realiza un estudio empírico, cuyo objetivo es proponer un procedimiento que permita visualizar dichos factores, sobre los cuales las empresas deben basar su planificación a corto y largo plazo.

Lo que se procura es determinar los factores de mayor incidencia en el desempeño financiero de las empresas, y que nos permiten distinguir entre empresas eficientes que son competitivas y empresas riesgosas que tienen dificultades financieras. Por tanto, el trabajo se enfoca en explicar el grado de correspondencia que tiene cada uno de los indicadores financieros en la rentabilidad económica.

En el presente trabajo se realiza, análisis de correlación en base a una muestra seleccionada compuesta por empresas del sector manufacturero de la provincia del Tungurahua que de acuerdo a la información obtenida del portal Web de 
la Superintendencia de Compañías se encuentran activas. El análisis correlación es una técnica de dependencia, cuyo objetivo es explicar o predecir un fenómeno, definido por una variable que actúa como dependiente en función de una serie de factores que se relacionan con él y que constituyen las variables independientes o explicativas. Para elegir estas últimas se dispone la base de datos de indicadores financieros.

Para identificar los factores determinantes de la rentabilidad se utiliza el coeficiente de correlación de PEARSON, al respecto, Rodríguez y Rodríguez,(2016) mencionan que en la correlación simple el valor de una variable independiente se emplea para predecir el valor de la varia dependiente. Se obtienen resultados entre -1 y +1 , mientras más se aproxima a +1 tiene una fuerte correlación positiva, mientras más se aproxima a -1 tiene una fuerte correlación negativa. Si el resultado es 0 implica ausencia de correlación.

Para cumplir con el objetivo de la investigación se ha tomado como muestra 140 sociedades de la provincia de Tungurahua. Para ello, se debe definir la variable dependiente que en este caso es el indicador de rentabilidad sobre el activo, así como también los indicadores que explican su contribución con la rentabilidad y que se consideran como variables independientes.

La base de datos utilizada para identificar los factores determinantes en la rentabilidad fue extraída del portal web de la Superintendencia de Compañías y depurada de acuerdo a los requerimientos del análisis. La base de datos contiene los valores de 20 indicadores financieros para cada compañía

\section{PROCEDIMIENTO}

Para identificar los factores determinantes de la rentabilidad de acuerdo a la base de datos disponible se efectuó el análisis por fases:

\section{FASE 1. DEPURACIÓN DE LA BASE DE DATOS.}

Para depurar la base de datos se obtuvo el promedio y la desviación estándar de los distintos indicadores, con el fin de identificar comportamientos inusuales, encontrándose que el indicador "rotación de cartera" era influido en forma significativa por los valores extremos, por ello se procedió a excluir del análisis a las compañías que presentaban indicadores negativos o muy altos en la rotación de cartera.

\section{FASE 2. CÁLCULO DE LOS COEFICIENTES DE CORRELACIÓN.}

Con la base de datos depurada se calcularon los coeficientes de correlación entre cada uno de los indicadores y el indicador de rentabilidad sobre el activo que permitió identificar los factores determinantes en la rentabilidad.

\section{RESULTADOS}

La aplicación del análisis de correlación de PEARSON, permitió calcular el grado de influencia de los diferentes indicadores en la rentabilidad sobre el activo (ROA) Obteniendo la tabla 1.

En el año 2014 el mayor impacto positivo corresponde al indicador de rotación de ventas con el 31,02\% y el menor impacto positivo corresponde al indicador de endeudamiento sobre el patrimonio con 0,33\%. Mientras que la correlación negativa más significativa es la de liquidez corriente con $-4,90 \%$. 
Tabla 1. Coeficientes de correlación de los indicadores con el roa en los años 2014 y 2015

\begin{tabular}{|l|c|c|}
\hline \multicolumn{1}{|c|}{ INDICADOR } & \multicolumn{1}{c|}{$\begin{array}{r}\text { ROE } \\
2014\end{array}$} & \multicolumn{1}{c|}{ ROE } \\
& 2015 \\
\hline LIQUIDEZ_CORRIENTE & $-4,90 \%$ & $5,20 \%$ \\
\hline PRUEBA_ACIDA & $-3,12 \%$ & $9,14 \%$ \\
\hline $\begin{array}{l}\text { ENDEUDAMIENTO_ } \\
\text { ACTIVO }\end{array}$ & $12,90 \%$ & $-20,01 \%$ \\
\hline $\begin{array}{l}\text { ENDEUDAMIENTO_ } \\
\text { PATRIMONIO }\end{array}$ & $0,33 \%$ & $-19,67 \%$ \\
\hline $\begin{array}{l}\text { ENDEUDAMIENTO_ } \\
\text { ACTIVO_FIJO_NETO }\end{array}$ & $0,53 \%$ & $5,45 \%$ \\
\hline APALANCAMIENTO & $0,34 \%$ & $-19,55 \%$ \\
\hline $\begin{array}{l}\text { APALANCAMIENTO_ } \\
\text { FINANCIERO }\end{array}$ & $2,01 \%$ & $-12,39 \%$ \\
\hline ROTACION_CARTERA & $-4,02 \%$ & $-9,55 \%$ \\
\hline $\begin{array}{l}\text { ROTACION_ACTIVO_ } \\
\text { FIJO }\end{array}$ & $-0,50 \%$ & $-1,09 \%$ \\
\hline ROTACION_VENTAS & $31,02 \%$ & $40,58 \%$ \\
\hline MARGEN_BRUTO & $15,38 \%$ & $7,93 \%$ \\
\hline
\end{tabular}

Fuente: Superintendencia de compañías del Ecuador Elaborado por: GMSM, 2017
En el año 2015 el mayor impacto positivo corresponde al indicador de rotación de ventas con el 40,58\% y el menor impacto positivo corresponde al indicador de liquidez corriente con el 5,20\%. Mientras que la correlación negativa más significativa corresponde al indicador de apalancamiento operático con $-19,55 \%$ Estos resultados se visualizan en la figura 1.

Además se realizó el análisis de correlación de la rentabilidad sobre el patrimonio también conocida como rentabilidad financiera o ROE, cuyos resultados se reflejan en la tabla 2

En el año 2014 el mayor impacto positivo corresponde al indicador de apalancamiento operativo con el 31,93\% y el menor impacto positivo corresponde al indicador de endeudamiento sobre el activo fijo neto con el 0,05\%. Mientras que la correlación negativa más significativa es la del margen bruto con $-8,79 \%$.

Figura 1. Coeficientes de Correlación de los indicadores financieros con el ROA en los años 2014 y 2015.

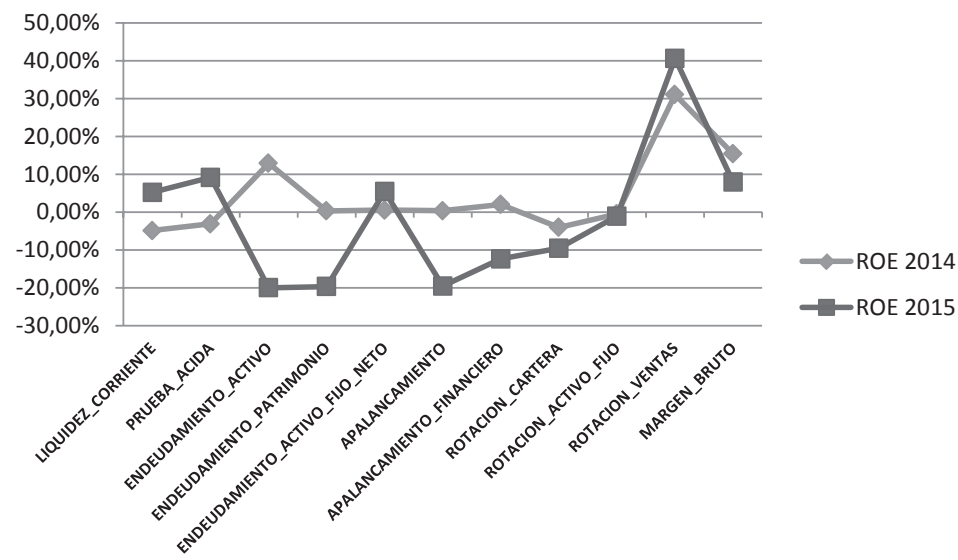

Fuente: Superintendencia de compañías del Ecuador Elaborado por: GMSM, 2017 
Tabla 2. Coeficientes de correlación de los indicadores con el roe en los años 2014 y 2015

\begin{tabular}{|l|r|r|}
\hline \multicolumn{1}{|c|}{ INDICADOR } & ROE 2014 & ROE 2015 \\
\hline $\begin{array}{l}\text { LIQUIDEZ_ } \\
\text { CORRIENTE }\end{array}$ & $-5,35 \%$ & $-6,42 \%$ \\
\hline PRUEBA_ACIDA & $-3,13 \%$ & $-4,92 \%$ \\
\hline $\begin{array}{l}\text { ENDEUDAMIENTO_ } \\
\text { ACTIVO }\end{array}$ & $10,80 \%$ & $6,33 \%$ \\
\hline $\begin{array}{l}\text { ENDEUDAMIENTO_ } \\
\text { PATRIMONIO }\end{array}$ & $43,96 \%$ & $3,87 \%$ \\
\hline $\begin{array}{l}\text { ENDEUDAMIENTO_ } \\
\text { ACTIVO_FIO_NETO }\end{array}$ & $0,05 \%$ & $-2,11 \%$ \\
\hline APALANCAMIENTO & $43,97 \%$ & $3,94 \%$ \\
\hline $\begin{array}{l}\text { APALANCAMIEN- } \\
\text { TO_FINANCIERO }\end{array}$ & $-3,91 \%$ & $0,68 \%$ \\
\hline ROTACION_CARTERA & $-2,52 \%$ & $-1,72 \%$ \\
\hline $\begin{array}{l}\text { ROTACION_ACTIVO_ } \\
\text { FIJO }\end{array}$ & $0,99 \%$ & $-1,39 \%$ \\
\hline ROTACION_VENTAS & $30,45 \%$ & $8,99 \%$ \\
\hline $\begin{array}{l}\text { RENTABILIDAD_ } \\
\text { NETA_ACTIVO }\end{array}$ & $23,19 \%$ & $31,93 \%$ \\
\hline MARGEN_BRUTO & $-8,79 \%$ & $9,14 \%$ \\
\hline
\end{tabular}

Fuente: Superintendencia de compañías del Ecuador Elaborado por: GMSM, 2017
En el año 2015 el mayor impacto positivo corresponde al indicador de rentabilidad neta sobre el activo con el $40,58 \%$ y el menor impacto positivo corresponde al indicador de apalancamiento financiero con el 0,68\%\%. Mientras que la correlación negativa más significativa corresponde a la liquidez corriente con el - 6,42\%. Estos resultados se visualizan en la figura 2

\section{DISCUSIÓN DE RESULTADOS}

Los resultados permitieron identificar claramente que el factor determinante de la rentabilidad sobre el activo (ROA) en las compañías manufactureras de la provincia del Tungurahua es la rotación de ventas, porque tanto en el año 2014 como en el año 2015 presentan una fuerte correlación. Es decir mientras las ventas roten a mayor velocidad mayor será la rentabilidad sobre el activo.

Mientras tanto la liquidez corriente tiene una incidencia inversa en la rentabilidad en el año 2014, lo cual confirma la teoría de que mayor liquidez genera menor

Figura 2. Coeficientes de Correlación de los indicadores financieros con el ROE en los años 2014 y 2015.

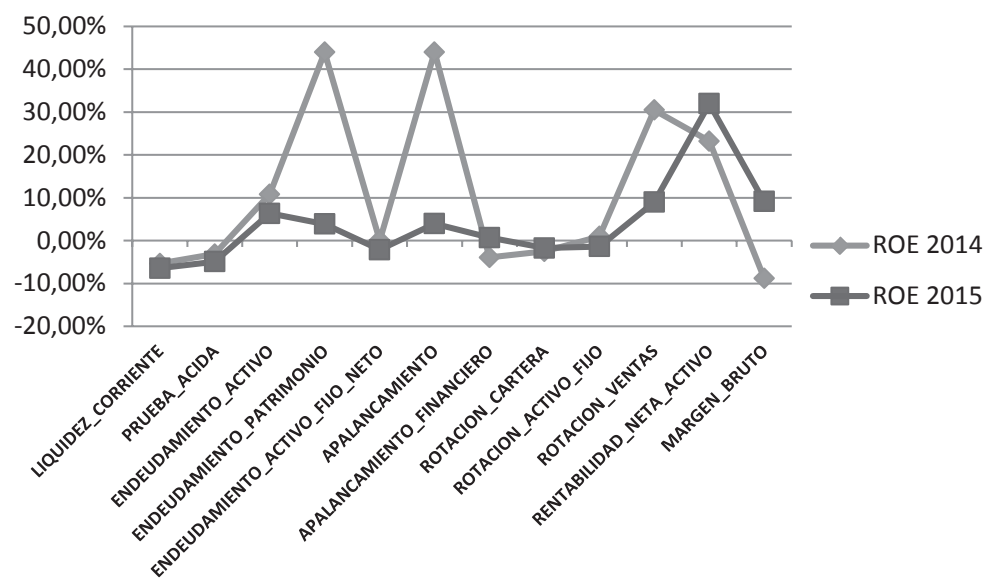

Fuente; Superintendencia de compañías del Ecuador Elaborado por: GMSM, 2017 
rentabilidad, sin embargo en el año 2015 existe una incidencia directa, ocasionada especialmente por la disminución de los indicadores de endeudamiento debido a la necesidad de mantener unos márgenes altos de liquidez para enfrentar la recesión económica que atraviesa el país.

En el ROE además de las rentabilidad neta sobre el activo también tienen correlación fuerte directa la rotación de ventas y el apalancamiento operativo. Mientras que la liquidez tiene una incidencia inversa. Los resultados reflejan la influencia del aprovechamiento de los costos fijos y la capacidad instalada en el desempeño empresarial.

\section{CONCLUSIONES}

1. El factor determinante en el desempeño financiero de las compañías del sector manufacturero de la provincia del Tungurahua, es la rotación de las ventas sobre el activo total. Es decir, mientras más veces representen las ventas con relación al activo la rentabilidad será mayor.

2. El análisis de correlación si permite identificar los factores determinantes del desempeño financiero en las compañías manufactureras de la provincia del Tungurahua.

3. Finalmente, es estudio permite recomendar como estrategia para mejorar la rentabilidad sobre el patrimonio, se debe mejorar el apalancamiento operativo mediante estrategias de diversificación o expansión. En caso de que el mercado no soporte la oferta se debe disminuir el tamaño de los activos fijos para mejorar los índices de competitividad y productividad.

\section{REFERENCIAS BIBLIOGRÁFICAS}

Castaño Ríos, C.E., Arias Pérez, J.E. (2014). Análisis financiero integral de empresas colombianas 20092012 desde la perspectiva dela competitividad, Revista U.D.C.A Actualidad \& Divulgación Científica 17.

Castillo Valero, Juan Sebastián y García Cortijo, María del Carmen (2012). Análisis de los factores explicativos de la rentabilidad de las empresas vinícolas de Castilla-La Mancha. Revista FCA.UNCUYO 45, 2013 Uncuyo, Argentina.

Estupiñán, R., y Estupiñán, O. (2006). Análisis finaciero y de gestión. Primera edición, ECOE EDICIONES, Bogotá.

Freeman, Christopher. (1998). La economia del cambio tecnologico. Recuperado de https://www.innova.uned.es/webpages/innovaciontecnologica/mod1_tema1/estudio17.pdf

IASC (1989). Framework for the Preparation and the Presentation of Financial Statements, IASC, London.

Kondratieff (1922) Las Ondas Largas. Editado por la Revista de la Facultad de Ciencias Económicas, UNMSM, 1990

Leontief, Wassily W. (1953). Domestic Production and Foreign Trade: The American Capital Position Re- examined. In Readings inn International Economics, edited by Richard E. Caves and Harry G. Johnson. Homewood, 1968.

Mazzei, Umberto. (2016). La crisis financiera mundial y el comercio. http://www.alainet.org/es/articulo/181078 
Medina Fernández del Soto, Jorge E (2010). Modelo integral de productividad, aspectos importantes para su implementación. Revista EAN No. 69 Julio-Diciembre 2010 Bogotá,

Ohlin, Bertil G. (1933). Interregional and International Trade. Cambridge, Mass: Harvard University Press.

Rivera Godoy, Jorge A.; Ruiz Acero, Daniel (2011. Análisis del desempeño financiero de empresas innovadoras del Sector Alimentos y Bebidas en Colombia. Pensamiento \& Gestión, núm. 31, 2011.

Robles Román, Carlos L. (2012). Fundamentos de administración financiera. Primera edición, Red tercer Milenio, México.

Rodríguez Franco, Jesús y Rodríguez Jiménez, Elba (2016). Estadística para Administración y economía. Segunda edición, Grupo Editorial Patria, México.

Schumpeter, Joseph. (1935). Análisis del cambio económico. En F. d. Económica, Ensayos sobre el ciclo económico. México: Fondo de Cultura Económica.

Van Horne J. y Wachowicz J.,(2010). Fundamentos de administración financiera. Décima Tercera edición, Pearson, México.

Wild J.; Subramanyam K.; y Halsey R., (2007). Análisis de Estados financieros. Novens edición, Mcgraw Hill, México.

\section{Para citaciones:}

Salazar-Mosquera, G. M. (2017). Factores determinantes del desempeño financiero en el sector manufacturero en la Republica del Ecuador. Panorama Económico, 25, 2, pp. 243-254.

\section{AUTOR}

